\title{
AN APPROXIMATION THEORY FOR OPTIMUM SHEETS IN UNILATERAL CONTACT
}

\author{
BY \\ JOAKIM PETERSSON (Dept. of Mechanical Engineering, Division of Mechanics, Linköping \\ Institute of Technology, Sweden) \\ AND
}

JAROSLAV HASLINGER (Dept. of Metal Physics, Faculty of Mathematics and Physics, Charles University, Prague, Czech Republic)

\begin{abstract}
In this paper we give an approximation theory for the optimum variable thickness sheet problem considered in [1] and [2]. This problem, which is a stiffness maximization of an elastic continuum in unilateral contact, admits complete material removal, i.e., the design variable is allowed to take zero values.

The original saddle-point problem is replaced by a sequence of approximating problems, the solutions of which are shown to converge weakly to exact solutions. In the case that complete material removal is not admissible, the state variable is shown to converge strongly in the $\left(H^{1}\right)^{2}$-norm to the unique exact state solution.

We consider a particular finite-element discretization that fits into the general theory and present the mathematical programming problem that results from it.
\end{abstract}

1. Introduction. In this paper we give a theory for how to obtain approximate solutions to the stiffest sheet problem. For a background to the structural optimization problem and its technological relevance, we refer to [3], [4], [1], [5], and [6].

Concerning qualitative analysis, Céa and Malanowski [7] gave in 1970 proofs of existence and uniqueness of solutions corresponding to non-contacting sheets and nonvanishing thickness. These results were generalized by Petersson [2] to include unilateral contact and zero lower bounds on designs, which hence turned the problem into a topology optimization problem.

Topology design of structures is a subject of widespread research [8], [9]. The optimum sheet problem, treated in this paper, differs from many of the investigated ones in topology optimization in the sense that it is well-posed in its original formulation, and need not be relaxed with homogenization techniques.

Received June 15, 1995.

1991 Mathematics Subject Classification. Primary 73K40, 65N30.

Key words and phrases. Optimum Sheet, Approximation Theory, Unilateral Contact, Topology Design. This work was supported by the Swedish Research Council for Engineering Sciences (TFR) and partially by grant No. 201/94/1863 of the Grant Agency of Czech Republic. 
We treat the continuous problem as well as its discretizations through saddle-point formulations. At first glance it seems that the situation is similar to the classical mixed finite-element approach [10]. But it is not so. In classical mixed finite-element methods, the saddle-point formulation results from releasing certain constraints by means of duality techniques. The Lagrange multiplier is in duality with the primal variable, in terms of which the original problem is formulated. Here the problem is rather different: the saddle-point formulation is the original problem and a consequence of the special choice of the cost functional; it has nothing to do with relaxing constraints. In fact, the variational problem is constrained in both variables, in contrast to classical saddle-point formulations.

Because of lack of uniqueness of the solution (at least of the thickness) and the fact that we cannot expect any regularity of it, we cannot expect that the classical LadyzhenskayaBabuska-Brezzi (LBB) condition, which plays a crucial role in mixed finite-element analyses, holds true in our case. We focus only on the convergence analysis. The scheme of the proof is standard: for the two finite-element spaces involved, we separately need to ensure the density and weak closedness properties. What is not standard is to choose the right topology in the case of finite-element spaces approximating the thickness.

The organization of the paper is the following. In Sec. 2 standard mathematical notations are introduced and a condensed version of the problem description is given. Section 3 , the core of the paper, gives the general approximation theory and its convergence results. In the fourth section we present a finite-element model that fits into the abstract setting in Sec. 3, and in the fifth (and last) section we present the problem to solve in practice and give some other concluding remarks.

2. Problem description. Before we define the problem, we introduce the standard notation of function spaces used in the paper. By $H^{k}(\Omega)$ (integer $k \geq 0$ ) we denote the classical Sobolev space of functions, derivatives of which up to the order $k$ are square integrable in $\Omega$ (i.e., elements of $L^{2}(\Omega)$ ). If $V$ is a subspace of $\left(H^{k}(\Omega)\right)^{2}$ (the definition is given below), the strong convergence of a sequence $\left\{u_{n}\right\}$ to $u, u_{n}, u \in V$ will be denoted by $u_{n} \stackrel{V}{\rightarrow} u$, while $u_{n} \stackrel{w}{\rightarrow} u$ stands for the weak convergence in $V$. By $L^{1}(\Omega), L^{\infty}(\Omega)$ we denote the spaces of Lebesgue integrable functions and essentially bounded measurable functions in $\Omega$, respectively. If $h_{n}, h \in L^{\infty}(\Omega)$ we write

$$
\begin{gathered}
h_{n} \stackrel{w^{*}}{\longrightarrow} h \text { in } L^{\infty}(\Omega) \text { iff } \int_{\Omega} h_{n} \varphi d \Omega \rightarrow \int_{\Omega} h \varphi d \Omega \quad \forall \varphi \in L^{2}(\Omega), \\
h_{n} \stackrel{\text { a.e. }}{\longrightarrow} h \text { iff } h_{n}(x) \rightarrow h(x) \quad \text { for almost all } x \in \Omega .
\end{gathered}
$$

We give a summary of the problem description and mathematical setting of the problem of optimizing a sheet in unilateral contact [2], [6].

Consider a sheet with a mid-surface occupying a domain $\Omega$ in $\mathbf{R}^{2}$, and with a thickness $h(x)$ at the point $x$ in $\Omega$. The boundary is partitioned into the parts $\Gamma_{u}, \Gamma_{t}$, and $\Gamma_{c}$, the first two denoting parts where zero displacements and tractions are prescribed, and the last meaning the candidate contact boundary; cf. Fig. 1. 


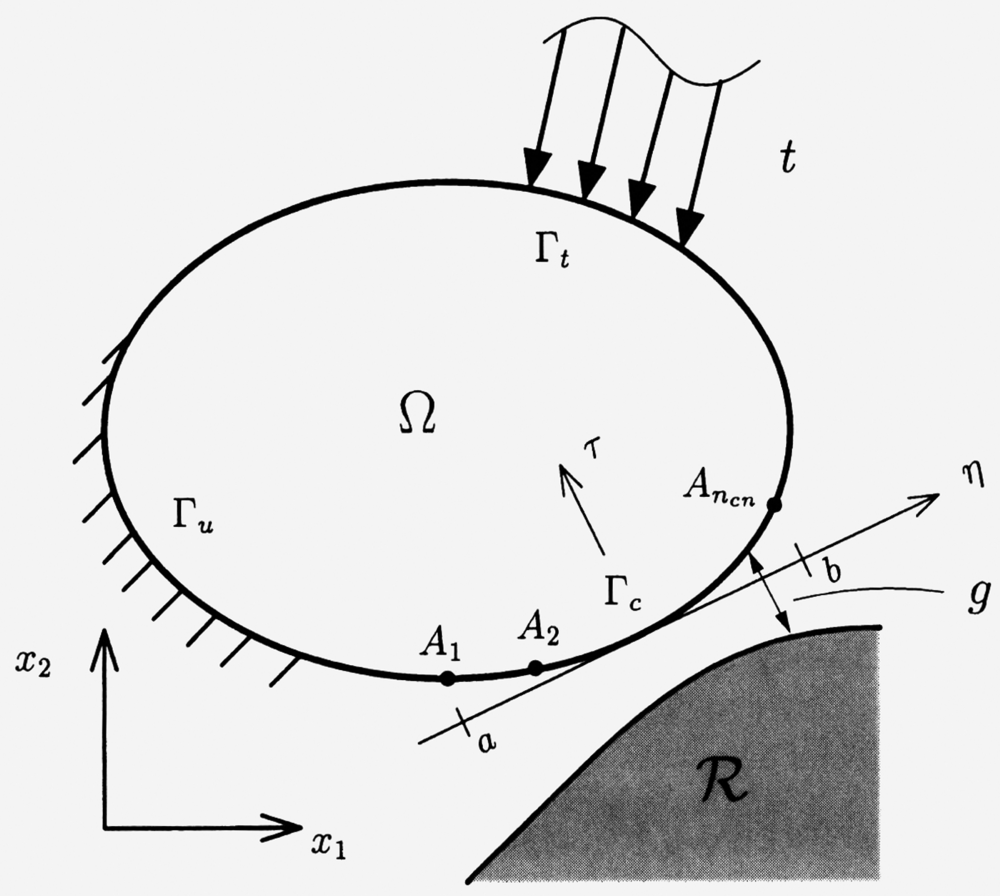

Fig. 1. The considered elastic body with possible unilateral contact

Each point $x$ in $\Omega$ can be assigned an in-plane displacement $u(x)$, and the corresponding field belongs to

$$
V=\left\{u=\left(u_{1}, u_{2}\right)^{\mathrm{T}} \in\left(H^{1}(\Omega)\right)^{2} \mid u_{i}=0 \text { on } \Gamma_{u}, i=1,2\right\} .
$$

Here $u_{i}$ denotes the displacement along the $x_{i}$-direction. We equip $V$ with the norm $\|\cdot\|_{V}$ :

$$
\|u\|_{V}=\left\{\int_{\Omega}\left(u_{i, j} u_{i, j}+u_{i} u_{i}\right) d \Omega\right\}^{1 / 2}=\sqrt{(u, u)_{V}}
$$

where $(\cdot, \cdot)_{V}$ is the inner product in $V$. (The summation convention in which repeated indices take the values 1 and 2 was used above, as will be sparsely done later at apparent places.) The considered contact is with a rigid frictionless obstacle occupying the domain $\mathcal{R}$, and the convex set of kinematically admissible displacements is denoted by $\mathcal{U}$, and defined through

$$
u(x)^{\mathrm{T}} n(x) \leq g(x) \quad \text { a.e. } x \in \Gamma_{c}
$$

where $n(x)$ is a normal vector of unit length, and $g(x)$ is a distance between $\Gamma_{c}$ and $\partial \mathcal{R}$ along the direction $n(x)$. This is a linearization of a more general large deformation nonpenetration condition [11], which can be done in different ways, leading to, e.g., $n=n^{\Omega}$ or $n=-n^{\mathcal{R}}$. (Here $n^{\mathcal{B}}$ denotes the outward unit normal to a region $\mathcal{B}$.) We mention that the first choice was used in [12] and the other in [13]. In this paper we follow an approach that is something in between these two choices, namely $n(x) \equiv \xi$, where $\xi \in \mathbf{R}^{2}$ is a fixed 
direction (opposite to the one indicated by the $\tau$-axis in Fig. 1). This was introduced in [14] and shown there to provide some mathematical conveniences. In order to guarantee the physical admissibility of a fixed direction, one has to exclude situations where the contacting boundaries are strongly curved. In any case, small deformations and linear elasticity imply that the above choices of $n$ are approximately equal [11]. A special case is when $\Gamma_{c}(\partial \mathcal{R})$ is a straight line, in which case $\xi$ coincides with $n^{\Omega}\left(-n^{\mathcal{R}}\right)$. In conclusion, we write

$$
\mathcal{U}=\left\{u \in V \mid u(x)^{\mathrm{T}} \xi \leq g(x) \text { a.e. } x \in \Gamma_{c}\right\}
$$

The total potential energy functional is given by

$$
L^{\infty}(\Omega) \times\left(H^{1}(\Omega)\right)^{2} \ni(h, u) \mapsto J_{h}(u) \in \mathbf{R},
$$

where

$$
J_{h}(u)=\frac{1}{2} a_{h}(u, u)-L(u)
$$

and where the linear and bilinear forms are given by

$$
L(u)=\int_{\Omega} f^{\mathrm{T}} u d \Omega+\int_{\Gamma_{t}} t^{\mathrm{T}} u d \Gamma \quad \text { and } \quad a_{h}(u, v)=\int_{\Omega} h \frac{\partial u_{i}}{\partial x_{j}} E_{i j k l} \frac{\partial v_{k}}{\partial x_{l}} d \Omega .
$$

Here $E_{i j k l}$ are the elasticity constants satisfying the usual ellipticity, symmetry, and boundedness conditions, $f \in\left(L^{2}(\Omega)\right)^{2}$ and $t \in\left(L^{2}\left(\Gamma_{t}\right)\right)^{2}$.

The set of admissible designs is defined as

$$
\mathcal{H}=\left\{h \in L^{\infty}(\Omega) \mid h_{\min } \leq h \leq h_{\max } \text { a.e. in } \Omega, \int_{\Omega} h d \Omega=V o l\right\},
$$

where $h_{\min }$ and $h_{\max }$ are bounding functions satisfying $0 \leq h_{\min } \leq h_{\max }, h_{\min }, h_{\max } \in$ $L^{\infty}(\Omega)$. The bounding function $h_{\min }$ can take zero values and $V o l$ is the available volume of material to be distributed in the domain $\Omega$.

The structural optimization problem to solve can now be formulated as

$$
(\mathrm{SJ})\left\{\begin{array}{l}
\text { Find }(\tilde{u}, \tilde{h}) \in \mathcal{U} \times \mathcal{H}: \\
J_{h}(\tilde{u}) \leq J_{\tilde{h}}(\tilde{u}) \leq J_{\tilde{h}}(u) \quad \forall(u, h) \in \mathcal{U} \times \mathcal{H}
\end{array}\right.
$$

Such a solution $(\tilde{u}, \tilde{h})$ gives a maximum of equilibrium potential energy and solves

$$
\min _{h \in \mathcal{H}} L(u(h))+\int_{\Gamma_{c}}\left|\sigma_{\xi}(u(h))\right| h g d \Gamma
$$

where $\sigma_{\xi}(u)=E_{i j k l} \frac{\partial u_{k}}{\partial x_{l}} \xi_{i} \xi_{j}$ and $u(h) \in \mathcal{U}$ solves

$$
a_{h}(u(h), \varphi-u(h)) \geq L(\varphi-u(h)) \quad \forall \varphi \in \mathcal{U} .
$$

There exist solutions to (SJ) as is shown in [2], but concerning uniqueness, the only one to expect is in $u$, in the case that the lower bound $h_{\min }$ is strictly positive. The set of solutions of (SJ) is bounded, weakly $\left({ }^{*}\right)$ closed, and convex. 


\section{A general approximation theory.}

3.1. Preliminaries. We want to approximate a solution $(\tilde{u}, \tilde{h})$ to (SJ). Suppose that $\left\{\mathcal{T}_{\rho_{m}}\right\}_{m=1}^{\infty}$ is any regular sequence of triangulations of $\bar{\Omega}$, where the mesh size parameter $\rho_{m}$ in the $m$ th triangulation (that is, the largest edge length of all triangles in $\mathcal{T}_{\rho_{m}}$ ) satisfies

$$
\rho_{m} \rightarrow 0 \quad(m \rightarrow \infty)
$$

For the definition of triangulation and regular families of such, we refer to [15]. In step $m, \mathcal{U}_{m} \subset V$ denotes the approximation of $\mathcal{U}$ and $\mathcal{H}_{m} \subset L^{\infty}(\Omega)$ ditto for $\mathcal{H}$. We make the following abstract assumptions:

(i) $\mathcal{H}_{m} \subset \mathcal{H} \quad \forall m$.

(ii) There is an interpolation operator $\Pi_{m}: \mathcal{H} \rightarrow \mathcal{H}_{m}$ such that $\Pi_{m} h \rightarrow h$ in $L^{1}(\Omega)$ as $m \rightarrow \infty, \quad \forall h \in \mathcal{H}$.

(j) For any sequence $\left\{u_{m}\right\}$ such that $u_{m} \in \mathcal{U}_{m}$ and $u_{m} \stackrel{w}{\rightarrow} u$ in $V$, it follows that $u \in \mathcal{U}$.

(jj) There exists a set $\chi \subset \mathcal{U}$ such that $\bar{\chi}=\mathcal{U}$ (the closure taken with respect to the $V$-norm) and there is an interpolation operator $\pi_{m}: \chi \rightarrow \mathcal{U}_{m}$ such that $\pi_{m} u \stackrel{V}{\rightarrow} u$ (in $\left.V\right)$ as $m \rightarrow \infty, \quad \forall u \in \chi$.

REMARK 3.1. From (jj) we conclude that, given any sequence $\left\{\rho_{m}\right\}$ and any $u \in \mathcal{U}$, there is a subsequence $\left\{\rho_{m_{i}}\right\}$ and $u_{i} \in \mathcal{U}_{m_{i}}$ such that $\left\|u_{i}-u\right\|_{V} \rightarrow 0$ as $i \rightarrow \infty$. Similarly, (ii) implies that, given any sequence $\left\{\rho_{m}\right\}$ and any $h \in \mathcal{H}$, there is a subsequence $\left\{\rho_{m_{i}}\right\}$ and $h_{i} \in \mathcal{H}_{m_{i}}$ such that $h_{i} \stackrel{\text { a.e. }}{\longrightarrow} h$ as $i \rightarrow \infty$.

An element $h_{0} \in \mathcal{H}$ is called a "coercive design" if there exists $\bar{\alpha}>0$ such that $a_{h_{0}}(u, u) \geq \bar{\alpha}\|u\|_{V}^{2}$ for all $u \in V$. In connection to this, we also assume

(k) There exists a coercive design $h_{0} \in \mathcal{H}$ such that $\Pi_{m} h_{0} \stackrel{L^{\infty}(\Omega)}{\longrightarrow} h_{0}$ as $m \rightarrow \infty$.

We will consider the approximating problems

$$
(\mathrm{SJ})_{m}\left\{\begin{array}{l}
\text { Find }\left(\tilde{u}_{m}, \tilde{h}_{m}\right) \in \mathcal{U}_{m} \times \mathcal{H}_{m}: \\
J_{h_{m}}\left(\tilde{u}_{m}\right) \leq J_{\tilde{h}_{m}}\left(\tilde{u}_{m}\right) \leq J_{\tilde{h}_{m}}\left(u_{m}\right) \quad \forall u_{m} \in \mathcal{U}_{m}, \forall h_{m} \in \mathcal{H}_{m},
\end{array}\right.
$$

for $m=1,2,3, \ldots$. We assume that this problem is solvable for each $m$. First, before stating the main result, two lemmas concerning the needed "tri-continuity" of $(h, u, v) \mapsto$ $a_{h}(u, v)$ will be established. Before stating them, note that by the Hölder inequality and boundedness of $E_{i j k l}$, one has

$$
\left|a_{h}(u, v)\right| \leq M\|h\|_{L^{\infty}(\Omega)}\|u\|_{V}\|v\|_{V},
$$

for some constant $M$ independent of $h \in L^{\infty}(\Omega)$ and $u, v \in V$.

LEMMA 3.1. Suppose that $h_{m} \stackrel{w^{*}}{\longrightarrow} h$ in $L^{\infty}(\Omega)\left(h_{m}, h \in \mathcal{H}\right)$ and $u_{m} \stackrel{V}{\rightarrow} u$ in $V\left(u_{m} \in\right.$ $\left.\mathcal{U}_{m}, u \in \mathcal{U}\right)$. The it follows that

$$
\lim _{m \rightarrow \infty} J_{h_{m}}\left(u_{m}\right)=J_{h}(u) .
$$

Proof. Clearly $L\left(u_{m}\right) \rightarrow L(u)$, and $a_{h_{m}}\left(u_{m}, u_{m}\right) \rightarrow a_{h}(u, u)$ is shown by standard arguments. 
LEMma 3.2. Suppose that $h_{m} \stackrel{\text { a.e. }}{\rightarrow} h\left(h_{m} \in \mathcal{H}_{m}, h \in \mathcal{H}\right)$, and $u_{m} \stackrel{w}{\rightarrow} u$ in $V\left(u_{m} \in\right.$ $\left.\mathcal{U}_{m}, u \in \mathcal{U}\right)$. Then, for any fixed $v \in V$,

$$
\lim _{m \rightarrow \infty} a_{h_{m}}\left(u_{m}, v\right)=a_{h}(u, v)
$$

and, moreover,

$$
\liminf _{m \rightarrow \infty} J_{h_{m}}\left(u_{m}\right) \geq J_{h}(u) .
$$

In the proof below we use a notation without implicit summations.

Proof. We have

$$
a_{h_{m}}\left(u_{m}, v\right)=\sum_{i, j, k, l} \int_{\Omega} h_{m} E_{i j k l} \frac{\partial\left(u_{m}\right)_{i}}{\partial x_{j}} \frac{\partial v_{k}}{\partial x_{l}} d \Omega \stackrel{\text { def }}{=} \sum_{i, j, k, l} I_{i j k l} .
$$

Set $t_{i j k l}=E_{i j k l} \partial v_{k} / \partial x_{l} \in L^{2}(\Omega)$, and study

$$
I_{i j k l}-\int_{\Omega} h t \frac{\partial u_{i}}{\partial x_{j}} d \Omega=\int_{\Omega}\left(h_{m} t \frac{\partial\left(u_{m}\right)_{i}}{\partial x_{j}}-h t \frac{\partial u_{i}}{\partial x_{j}}\right) d \Omega
$$

(we omit the subscripts of $t$ ). We rewrite (7) as

$$
I_{i j k l}-\int_{\Omega} h t \frac{\partial u_{i}}{\partial x_{j}} d \Omega=\int_{\Omega}\left(\frac{\partial\left(u_{m}\right)_{i}}{\partial x_{j}}-\frac{\partial u_{i}}{\partial x_{j}}\right) h t d \Omega+\int_{\Omega}\left(h_{m}-h\right) \frac{\partial\left(u_{m}\right)_{i}}{\partial x_{j}} t d \Omega .
$$

The first integral on the right-hand side in (8) tends to zero as $m \rightarrow \infty$. Let us study the second term:

$$
\left|\int_{\Omega}\left(h_{m}-h\right) \frac{\partial\left(u_{m}\right)_{i}}{\partial x_{j}} t d \Omega\right|^{2} \leq \int_{\Omega}\left(h_{m}-h\right)^{2} t^{2} d \Omega \cdot \int_{\Omega}\left(\frac{\partial\left(u_{m}\right)_{i}}{\partial x_{j}}\right)^{2} d \Omega .
$$

In order to arrive at (5) it is now sufficient to show that

$$
\lim _{m \rightarrow \infty} \int_{\Omega}\left(h_{m}-h\right)^{2} t^{2} d \Omega=0 .
$$

This follows from Lebesgue's theorem of dominated convergence.

The second part of the proof is devoted to (6). Since $h_{m}$ is nonnegative and $a_{h_{m}}$ is symmetric we have

$$
0 \leq a_{h_{m}}\left(u_{m}-u, u_{m}-u\right)=a_{h_{m}}\left(u_{m}, u_{m}\right)+a_{h_{m}}(u, u)-2 a_{h_{m}}\left(u_{m}, u\right) .
$$

Hence, by rearranging terms,

$$
a_{h_{m}}\left(u_{m}, u_{m}\right) \geq 2 a_{h_{m}}\left(u_{m}, u\right)-a_{h_{m}}(u, u),
$$

from which

$$
\liminf _{m \rightarrow \infty} a_{h_{m}}\left(u_{m}, u_{m}\right) \geq a_{h}(u, u)
$$

follows by using (5). Clearly,

$$
\lim _{m \rightarrow \infty} L\left(u_{m}\right)=L(u)
$$

and (13) and (14) imply (6). 


\subsection{Convergence results. The main abstract result is}

Theorem 3.1. Let all abstract assumptions (i), (ii), (j), (jj), and (k) be satisfied. Suppose that $\left\{\mathcal{T}_{\rho_{m}}\right\}_{m=1}^{\infty}$ is any regular sequence of triangulations with a corresponding sequence of mesh size parameters $\left\{\rho_{m}\right\}_{m=1}^{\infty}$ obeying (3). Suppose further that $\left(\tilde{u}_{m}, \tilde{h}_{m}\right) \in \mathcal{U}_{m} \times \mathcal{H}_{m}$ solves $(\mathrm{SJ})_{m}$ for $m=1,2,3, \ldots$ Then there are subsequences $\left\{\tilde{u}_{m_{i}}\right\},\left\{\tilde{h}_{m_{i}}\right\}$ and elements $\tilde{u} \in \mathcal{U}, \tilde{h} \in \mathcal{H}$ such that

$$
\tilde{u}_{m_{i}} \stackrel{w}{\rightarrow} \tilde{u} \text { in } V, \quad \tilde{h}_{m_{i}} \stackrel{w^{*}}{\longrightarrow} \tilde{h} \text { in } L^{\infty}(\Omega) .
$$

Furthermore, any such pair of cluster points $(\tilde{u}, \tilde{h})$ solves (SJ).

Proof. Step 1. We estimate, in order, the third and first terms in the definition of $(\mathrm{SJ})_{m}$. Fix $u_{0} \in \chi$. We then have

$$
\begin{aligned}
J_{\tilde{h}_{m}}\left(\pi_{m} u_{0}\right) & =\frac{1}{2} a_{\tilde{h}_{m}}\left(\pi_{m} u_{0}, \pi_{m} u_{0}\right)-L\left(\pi_{m} u_{0}\right) \\
& \leq \frac{M}{2}\left\|\tilde{h}_{m}\right\|_{L^{\infty}(\Omega)}\left\|\pi_{m} u_{0}\right\|_{V}^{2}+C_{L}\left\|\pi_{m} u_{0}\right\|_{V},
\end{aligned}
$$

with the aid of (4). Now, due to (i) and the fact that $\left\{\pi_{m} u_{0}\right\}$ converges to $u_{0}$ in $V$, $\left\|\tilde{h}_{m}\right\|_{L^{\infty}(\Omega)}$ and $\left\|\pi_{m} u_{0}\right\|_{V}$ are bounded. This in (15) yields

$$
\exists C_{1}>0: \quad J_{\tilde{h}_{m}}\left(\pi_{m} u_{0}\right) \leq C_{1} \quad \forall m .
$$

Now, let $h_{0} \in \mathcal{H}$ be as in $(\mathbf{k})$. Then

$$
J_{\Pi_{m} h_{0}}\left(\tilde{u}_{m}\right)=\frac{1}{2} a_{h_{0}}\left(\tilde{u}_{m}, \tilde{u}_{m}\right)-L\left(\tilde{u}_{m}\right)+\frac{1}{2} a_{\Pi_{m} h_{0}-h_{0}}\left(\tilde{u}_{m}, \tilde{u}_{m}\right) .
$$

Using (4), (17), and the fact that $a_{h_{0}}$ is elliptic we have

$$
\begin{aligned}
J_{\Pi_{m} h_{0}}\left(\tilde{u}_{m}\right) & \geq \frac{\bar{\alpha}}{2}\left\|\tilde{u}_{m}\right\|_{V}^{2}-C_{L}\left\|\tilde{u}_{m}\right\|_{V}-\frac{M}{2}\left\|\Pi_{m} h_{0}-h_{0}\right\|_{L^{\infty}(\Omega)}\left\|\tilde{u}_{m}\right\|_{V}^{2} \\
& =\frac{1}{2}\left(\bar{\alpha}-M\left\|\Pi_{m} h_{0}-h_{0}\right\|_{L^{\infty}(\Omega)}\right)\left\|\tilde{u}_{m}\right\|_{V}^{2}-C_{L}\left\|\tilde{u}_{m}\right\|_{V} .
\end{aligned}
$$

Since $\left\|\Pi_{m} h_{0}-h_{0}\right\|_{L^{\infty}(\Omega)} \rightarrow 0,(18)$ implies that for all $m$ sufficiently large

$$
\begin{aligned}
J_{\Pi_{m} h_{0}}\left(\tilde{u}_{m}\right) & \geq \frac{\bar{\alpha}}{4}\left\|\tilde{u}_{m}\right\|_{V}^{2}-C_{L}\left\|\tilde{u}_{m}\right\|_{V} \\
& =\left(\frac{\sqrt{\bar{\alpha}}}{2}\left\|\tilde{u}_{m}\right\|_{V}-\frac{C_{L}}{\sqrt{\bar{\alpha}}}\right)^{2}-C_{L}^{2} \bar{\alpha}^{-1} \\
& \geq-C_{L}^{2} \bar{\alpha}^{-1} .
\end{aligned}
$$

Now $(\mathrm{SJ})_{m},(16)$, and (19) show that $\left\{J_{\tilde{h}_{m}}\left(\tilde{u}_{m}\right)\right\}$ is bounded in $\mathbf{R}$ and $\left\{\tilde{u}_{m}\right\}$ is bounded in $V$.

Step 2. One can extract subsequences $\left\{\tilde{h}_{m_{i}}\right\},\left\{\tilde{u}_{m_{i}}\right\}$, and $\left\{J_{\tilde{h}_{m_{i}}}\left(\tilde{u}_{m_{i}}\right)\right\}$ such that $\tilde{h}_{m_{i}}$ tends $L^{\infty}(\Omega)$ weakly* to a limit, say $\tilde{h}, \tilde{u}_{m_{i}}$ weakly in $V$ to a limit called $\tilde{u}$, and furthermore $J_{\tilde{h}_{m_{i}}}\left(\tilde{u}_{m_{i}}\right)$ converges to a real number $\kappa$. The set $\mathcal{H}$ is $L^{\infty}(\Omega)$ weakly* closed; so (i) implies that $\tilde{h} \in \mathcal{H}$, and from $(\mathbf{j})$ we infer $\tilde{u} \in \mathcal{U}$. To conclude, we have

$$
J_{\tilde{h}_{m_{i}}}\left(\tilde{u}_{m_{i}}\right) \rightarrow \kappa, \quad \tilde{h}_{m_{i}} \stackrel{w^{*}}{\longrightarrow} \tilde{h} \in \mathcal{H} \text { in } L^{\infty}(\Omega), \quad \tilde{u}_{m_{i}} \stackrel{w}{\rightarrow} \tilde{u} \in \mathcal{U} \text { in } V .
$$


Step 3. Let $u \in \mathcal{U}$ and $h \in \mathcal{H}$ be arbitrary. Choosing $h_{i} \in \mathcal{H}_{m_{i}}$ and $u_{i} \in \mathcal{U}_{m_{i}}$ as in Remark 3.1, $(\mathrm{SJ})_{m_{i}}$ reads

$$
J_{h_{i}}\left(\tilde{u}_{m_{i}}\right) \leq J_{\tilde{h}_{m_{i}}}\left(\tilde{u}_{m_{i}}\right) \leq J_{\tilde{h}_{m_{i}}}\left(u_{i}\right) .
$$

Applying Lemmas 3.1 and 3.2, we pass to the limit in (21):

$$
J_{h}(\tilde{u}) \leq \kappa \leq J_{\tilde{h}}(u) \quad \forall u \in \mathcal{U}, \quad \forall h \in \mathcal{H} .
$$

This means that $\kappa=J_{\tilde{h}}(\tilde{u})$ and consequently $(\tilde{u}, \tilde{h})$ solves (SJ).

In the case when "zero designs" are non-admissible, that is, when no topological information will be given by an optimal solution, we will show that the convergence properties are stronger. To this end, we need the following

LEMMA 3.3. Suppose that $\left(\tilde{u}_{m}, \tilde{h}_{m}\right)$ solves $(\mathrm{SJ})_{m}$ and that there are subsequences $\left\{\tilde{u}_{m_{i}}\right\}$, $\left\{\tilde{h}_{m_{i}}\right\}$ such that

$$
\tilde{u}_{m_{i}} \stackrel{w}{\rightarrow} \tilde{u} \text { in } V, \quad \tilde{h}_{m_{i}} \stackrel{w^{*}}{\longrightarrow} \tilde{h} \text { in } L^{\infty}(\Omega) \quad(i \rightarrow \infty),
$$

where $(\tilde{u}, \tilde{h})$ solves (SJ), i.e., the situation in Theorem 3.1. Then there is a subsequence of $\left\{\tilde{u}_{m_{i}}\right\}$, denoted again by $\left\{\tilde{u}_{m_{i}}\right\}$, and a sequence $\left\{h_{i}\right\}$ such that $h_{i} \in \mathcal{H}_{m_{i}}$ and

$$
\lim _{i \rightarrow \infty} a_{h_{i}}\left(\tilde{u}_{m_{i}}-\tilde{u}, \tilde{u}_{m_{i}}-\tilde{u}\right)=0 .
$$

Proof. Let $\left\{\tilde{u}_{m_{i}}\right\},\left\{\tilde{h}_{m_{i}}\right\}$ be sequences satisfying (23) and let $\left\{\rho_{m_{i}}\right\}$ be the corresponding family of partitions. According to Remark 3.1 it is possible to find a subsequence, again denoted by $\left\{\rho_{m_{i}}\right\}$, and elements $h_{i} \in \mathcal{H}_{m_{i}}, u_{i} \in \mathcal{U}_{m_{i}}$ such that $h_{i} \stackrel{\text { a.e. }}{\longrightarrow} \tilde{h}$ an $\left\|u_{i}-\tilde{u}\right\|_{V} \rightarrow 0$ as $i \rightarrow \infty$.

Choosing $u_{m_{i}}=u_{i}$ and $h_{m_{i}}=h_{i}$ in $(\mathrm{SJ})_{m_{i}}$, we obtain

$$
J_{h_{i}}\left(\tilde{u}_{m_{i}}\right) \leq J_{\tilde{h}_{m_{i}}}\left(u_{i}\right), \quad i=1,2,3, \ldots
$$

This can be rewritten as

$$
a_{h_{i}}\left(\tilde{u}_{m_{i}}, \tilde{u}_{m_{i}}\right) \leq a_{\tilde{h}_{m_{i}}}\left(u_{i}, u_{i}\right)+2 L\left(\tilde{u}_{m_{i}}-u_{i}\right) .
$$

Using (25) and the fact that the $h_{i}$ are nonnegative, we get

$$
\begin{aligned}
0 & \leq a_{h_{i}}\left(\tilde{u}_{m_{i}}-\tilde{u}, \tilde{u}_{m_{i}}-\tilde{u}\right) \\
& \leq a_{\tilde{h}_{m_{i}}}\left(u_{i}, u_{i}\right)+2 L\left(\tilde{u}_{m_{i}}-u_{i}\right)+a_{h_{i}}(\tilde{u}, \tilde{u})-2 a_{h_{i}}\left(\tilde{u}_{m_{i}}, \tilde{u}\right) .
\end{aligned}
$$

We have $\tilde{h}_{m_{i}} \stackrel{w^{*}}{\longrightarrow} \tilde{h}$ in $L^{\infty}(\Omega), \tilde{u}_{m_{i}} \stackrel{w}{\rightarrow} \tilde{u}$ in $V, h_{i} \stackrel{\text { a.e. }}{\longrightarrow} \tilde{h}$ and $u_{i} \stackrel{V}{\rightarrow} \tilde{u}$, and so, passing to the limit in (26), using Lemmas 3.1 and 3.2, one arrives at (24).

REMARK 3.2. From the proof of Lemma 3.3 it follows that (24) holds for any sequence $\left\{h_{i}\right\}, h_{i} \in \mathcal{H}_{m_{i}}$, such that $h_{i} \stackrel{\text { a.e. }}{\longrightarrow} \tilde{h}$.

We are now ready to state and prove the stronger convergence statement:

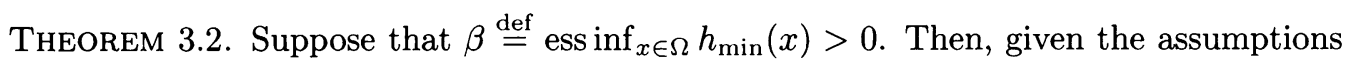
in Theorem 3.1, it follows that

$$
\left\|\tilde{u}_{m}-\tilde{u}\right\|_{V} \rightarrow 0 \quad(m \rightarrow \infty),
$$


that is, in addition to what is stated in Theorem 3.1 , the whole sequence $\left\{\tilde{u}_{m}\right\}$ tends strongly to $\tilde{u}$.

Proof. Any weak cluster point of $\left\{\tilde{u}_{m}\right\}$ is part of a solution to (SJ) and therefore unique due to Theorem 4.1 in [2] (assuming $\beta>0$ ). Hence $\tilde{u}_{m} \stackrel{w}{\rightarrow} \tilde{u}$ in $V$, i.e., the whole sequence converges to $\tilde{u}$ weakly in $V$. We show that for all subsequences $\left\{\tilde{u}_{m_{i}}\right\}$ of $\left\{\tilde{u}_{m}\right\}$, there is yet another subsequence, denoted again by $\left\{\tilde{u}_{m_{i}}\right\}$, which converges strongly in $V$ to $\tilde{u}$, and then (27) can be concluded.

Let $\left\{\tilde{u}_{m_{i}}\right\}$ be any subsequence of $\left\{\tilde{u}_{m}\right\}$. Then there are subsequences $\left\{\tilde{u}_{m_{i}}\right\},\left\{\tilde{h}_{m_{i}}\right\}$, $\tilde{u}_{m_{i}} \in \mathcal{U}_{m_{i}}, \tilde{h}_{m_{i}} \in \mathcal{H}_{m_{i}}$, and elements $h_{i} \in \mathcal{H}_{m_{i}}$ such that (23) holds. From Lemma 3.3 we therefore get (24). Since $\beta>0$ and, from (i), $h_{i} \in \mathcal{H}$, there exists $\bar{\alpha}>0$ such that for all $i$

$$
0 \leq \bar{\alpha}\left\|\tilde{u}_{m_{i}}-\tilde{u}\right\|_{V}^{2} \leq a_{h_{i}}\left(\tilde{u}_{m_{i}}-\tilde{u}, \tilde{u}_{m_{i}}-\tilde{u}\right),
$$

which together with (24) implies $\left\|\tilde{u}_{m_{i}}-\tilde{u}\right\|_{V} \rightarrow 0$, meaning that the proof is complete.

REMARK 3.3. For practical reasons it would also be possible to use two different partitions of $\bar{\Omega}$, one for the construction of the set $\mathcal{U}_{m}$, the other for $\mathcal{H}_{m}$. The convergence analysis can be done in a similar way as before.

4. A particular finite-element approximation. In this section we study a particular finite-element approximation. We will describe the discretization for a fixed $m$, sufficiently large for $(\mathrm{SJ})_{m}$ to be practically useful. To this end let us suppose that $\Omega$ is a plane polygonal domain that is subdivided into $n_{\mathrm{el}}$ number of triangles/elements $\bar{\Omega}^{e}$ such that $\bar{\Omega}=\bigcup_{e=1}^{n_{\mathrm{el}}} \bar{\Omega}^{e}$. We define the following finite element space:

$$
V_{m}=\left\{u_{m} \in(C(\bar{\Omega}))^{2}\left|u_{m}\right|_{\bar{\Omega}^{e}} \in\left(P_{1}\right)^{2}, e=1, \ldots, n_{\mathrm{el}}, u_{m}=0 \text { on } \bar{\Gamma}_{u}\right\},
$$

which is an internal approximation of $V . P_{1}$ denotes the space of linear polynomials (in two variables). By $\left\{N_{1}, \ldots, N_{n_{\mathrm{np}}}\right\}$ we denote the usual basis for the scalar counterpart of $V_{m} . N_{A}$ is referred to as a shape function in $V_{m}$ associated with the node number $A$, and $n_{\text {np }}$ denotes the number of nodal points (triangle vertices) in $\bar{\Omega} \backslash \bar{\Gamma}_{u}$. Any $u_{m}=$ $\left(u_{m}^{1}, u_{m}^{2}\right)^{\mathrm{T}} \in V_{m}$ has the representation

$$
u_{m}(x)=\sum_{A=1}^{n_{\mathrm{np}}} N_{A}(x) d_{A}
$$

where $d_{A}=\left(d_{1 A}, d_{2 A}\right)^{\mathrm{T}}$, and $d_{i A}$ is the value of $u_{m}^{i}$ at node number $A$. We let $\mathcal{M}: V_{m} \rightarrow$ $\mathbf{R}^{2 n_{\mathrm{np}}}$ be the isomorphism given by

$$
\mathcal{M} u_{m}=d=\left(d_{1}, \ldots, d_{2 n_{\mathrm{np}}}\right)^{\mathrm{T}},
$$

where $d$ is a collection of all the $d_{i A}$ 's such that each $d_{i A}$ appears exactly once in $d$ at a unique position. Suppose that $d_{1 A}$ is placed in the $I$ th position in $d$ and $d_{2 A}$ in the $J$ th. Let, for $A=1, \ldots, n_{\mathrm{np}}, C_{A}$ be the $2 n_{\mathrm{np}} \times 2$-matrix defined by

$$
\left(C_{A}\right)_{i j}= \begin{cases}1, & \text { if }(i, j)=(I, 1) \text { or }(i, j)=(J, 2), \\ 0, & \text { otherwise. }\end{cases}
$$


Then it follows that

$$
d_{A}=C_{A}^{\mathrm{T}} d .
$$

We let $n_{\mathrm{cn}}$ be the number of contact nodes, that is, the number of nodes in $\bar{\Gamma}_{c} \backslash \bar{\Gamma}_{u}$, and let $A_{i}$ denote the coordinate of the $i$ th contact node. We suppose that the gap function $g$ is pointwise defined.

As an approximation of $\mathcal{U}$ we take the set

$$
\mathcal{U}_{m}=\left\{u_{m} \in V_{m} \mid u_{m}\left(A_{i}\right)^{\mathrm{T}} \xi \leq g\left(A_{i}\right), i=1, \ldots, n_{\mathrm{cn}}\right\} .
$$

We approximate thickness functions as elementwise constant over $\mathcal{T}_{\rho_{m}}$, and therefore let $L_{m} \subset L^{\infty}(\Omega)$ be the closed subspace defined as the span of $\left\{\chi_{\Omega^{1}}, \ldots, \chi_{\Omega^{n}{ }^{n} \mathrm{l}}\right\}$, where $\chi_{\Omega^{e}}$ is the characteristic function of $\Omega^{e}$ :

$$
L_{m}=\left\{h_{m} \mid \exists t_{e} \in \mathbf{R}, e=1, \ldots, n_{\mathrm{el}}: h_{m}=\sum_{e=1}^{n_{\mathrm{el}}} t_{e} \chi_{\Omega^{e}} \text { a.e. in } \bar{\Omega}\right\} .
$$

Further, we define

$$
\mathcal{H}_{m}=L_{m} \cap \mathcal{H} .
$$

For the sake of simplicity we study cases when the design bound functions and elasticity coefficients are constant: $h_{\max } \equiv \alpha_{U}>0$ and $h_{\min } \equiv \alpha_{L} \geq 0$.

Similarly to $\mathcal{M}$, we define $\mathcal{N}$ to be the isomorphism from $L_{m}$ onto $\mathbf{R}^{n_{\text {el }}}$ with

$$
\mathcal{N} h_{m}=t=\left(t_{1}, \ldots, t_{n_{\mathrm{el}}}\right)^{\mathrm{T}} .
$$

To justify the described FE-model we simply need to verify (i), (ii), (j), (jj), and (k) of the general framework given in the previous section.

4.1. Verification of (i), (ii), and (k). From the definition of $\mathcal{H}_{m}$, i.e., (32), (i) follows immediately.

As the operator $\Pi_{m}$ we choose the elementwise constant interpolation

$$
\Pi_{m} h=\sum_{e=1}^{n_{\mathrm{el}}} t_{e} \chi_{\Omega^{e}},
$$

where $t_{e}$ is the integral mean of $h$ over $\Omega^{e}$,

$$
t_{e}=\frac{1}{S_{e}} \int_{\Omega^{e}} h d \Omega ; \quad S_{e}=\operatorname{meas}\left(\Omega^{e}\right) .
$$

It is a standard result of Lebesgue integration that $\Pi_{m} h \rightarrow h$ in $L^{1}(\Omega)$ for any $h$ in $\mathcal{H}$. Clearly $\Pi_{m} h \in \mathcal{H}_{m}$, and (ii) is hence verified.

To check $(\mathbf{k})$ one only has to take $h_{0} \equiv \mathrm{Vol} /$ meas $(\Omega)$, since $h_{\max }$ and $h_{\min }$ are constant, and note that $\Pi_{m} h_{0}=h_{0}$ for any $m$.

4.2. Verification of $(\mathbf{j})$. In order to specify the situation geometrically, let us assume that the contact part $\Gamma_{c}$ is described in the local coordinate system $(\eta, \tau)$ by the graph of a Lipschitz continuous function $\alpha:[a, b] \rightarrow \mathbf{R}^{1}$, i.e.,

$$
\bar{\Gamma}_{c}=\{(\eta, \tau) \mid \tau=\alpha(\eta), \eta \in[a, b]\},
$$

cf. Fig. 1. The non-penetration condition on $\Gamma_{c}$ now reads as follows:

$$
u_{\xi}(\eta, \alpha(\eta)) \equiv u(\eta, \alpha(\eta))^{\mathrm{T}} \xi \leq g(\eta, \alpha(\eta)), \quad \eta \in[a, b],
$$


where $g$ is the distance function between $\Gamma_{c}$ and the rigid foundation along the fixed direction $\xi \in \mathbf{R}^{2}$.

Let $A_{i}=\left(\eta_{i}, \alpha\left(\eta_{i}\right)\right)$ denote the coordinate of the $i$ th contact node in the local system, $i=1, \ldots, n_{\mathrm{cn}}$, where $a \equiv \eta_{1}<\eta_{2}<\cdots<\eta_{n_{\mathrm{cn}}} \equiv b$ is a partition of $[a, b]$.

We shall distinguish two cases:

$1^{\circ} . g(\cdot, \alpha(\cdot))$ is a concave function;

$2^{\circ} \cdot g(\cdot, \alpha(\cdot))$ is a general Lipschitz continuous function.

If $1^{\circ}$ is satisfied, then for any $u \in \mathcal{U}_{m}$,

$$
u_{\xi}\left(A_{i}\right) \leq g\left(A_{i}\right), \quad i=1, \ldots, n_{\mathrm{cn}} \Rightarrow u_{\xi}(\eta, \alpha(\eta)) \leq g(\eta, \alpha(\eta))
$$

for any $\eta \in[a, b]$. Thus $\mathcal{U}_{m}$ is an internal approximation of $\mathcal{U}$, i.e., $\mathcal{U}_{m} \subset \mathcal{U}$ for $m=1, \ldots$ Therefore, the condition $(\mathbf{j})$ is automatically satisfied.

Now, let us pass to the general case $2^{\circ}$. We shall need the following auxiliary result:

LEMma 4.1. Let $\varphi$ be a continuous function defined on $[a, b](-\infty<a<b<\infty)$, $D_{n}: a \equiv \eta_{0}^{n}<\eta_{1}^{n}<\cdots<\eta_{n}^{n} \equiv b$ be a partition of $[a, b]$, the norm $\nu\left(D_{n}\right)=\max _{i=1, \ldots, n} \mid \eta_{i}^{n}$ $-\eta_{i-1}^{n} \mid$ of which tends to zero as $n \rightarrow \infty$. Let $\left\{\psi_{n}\right\}_{n=1}^{\infty}$ be a sequence of piecewise linear functions over $\left\{D_{n}\right\}$ such that

$$
\psi_{n}\left(\eta_{i}^{n}\right) \geq \varphi\left(\eta_{i}^{n}\right), \quad i=0, \ldots, n, \quad \forall n .
$$

Let $\psi_{n} \stackrel{\text { a.e. }}{\longrightarrow} \psi, n \rightarrow \infty$, in $[a, b]$. Then $\psi \geq \varphi$ almost everywhere in $[a, b]$.

Proof. See [16], Lemma A2.

Lemma 4.2. Let $2^{\circ}$ be satisfied. Then the assumption (j) holds for any regular system of triangulations $\left\{\mathcal{T}_{\rho_{m}}\right\}$.

Proof. Let $\left\{u_{m}\right\}, u_{m} \in \mathcal{U}_{m}$ be such that

$$
u_{m} \stackrel{w}{\rightarrow} u \text { in } V .
$$

Then, by the compactness of the trace mapping, $u_{m} \rightarrow u$ in $\left(L^{2}\left(\Gamma_{c}\right)\right)^{2}$ and also

$$
u_{m}^{\mathrm{T}} \xi \rightarrow u^{\mathrm{T}} \xi \text { in } L^{2}\left(\Gamma_{c}\right) .
$$

As before, one can find a subsequence (still denoted by the same symbol) such that

$$
u_{m}^{\mathrm{T}} \xi \stackrel{\text { a.e. }}{\longrightarrow} u^{\mathrm{T}} \xi \quad \text { in }[a, b] .
$$

Moreover, $u_{m}^{\mathrm{T}} \xi$ is piecewise linear in $[a, b]$. The rest of the proof now follows immediately from Lemma 4.1 .

4.3. Verification of (jj). For the sake of simplicity, to avoid the manipulation of coordinate rotations, assume that the local system $(\eta, \tau)$ coincides with the cartesian system $\left(x_{1}, x_{2}\right)$. Let $s:[a, b] \rightarrow \mathbf{R}^{1}$ be the function that defines the boundary of the rigid obstacle $\mathcal{R}$ in a vicinity of $\Gamma_{c}$; cf. Fig. 2. Concerning the mutual position of $\Omega$ and $\mathcal{R}$ we also make the following geometrical assumption:

The function $s$ can be extended from $[a, b]$ to the whole $\mathbf{R}^{1}$ in such a way that its extension $\tilde{s}: \mathbf{R}^{1} \rightarrow \mathbf{R}^{1}$ exists with the following properties:

- $\left.\tilde{s}\right|_{[a, b]}=s$

- $\tilde{s}$ is sufficiently smooth in $\mathbf{R}^{1}$; 
- $\Gamma_{u} \subset \mathcal{Q} \equiv \mathcal{R}^{c}=\left\{\left(x_{1}, x_{2}\right) \mid x_{2} \geq \tilde{s}\left(x_{1}\right), x_{1} \in \mathbf{R}^{1}\right\}$.

The non-penetration condition now reads as follows:

$$
u_{2}\left(x_{1}, \alpha\left(x_{1}\right)\right) \geq-\alpha\left(x_{1}\right)+s\left(x_{1}\right) \quad \forall x_{1} \in(a, b),
$$

i.e., $g\left(x_{1}, \alpha\left(x_{1}\right)\right)=\alpha\left(x_{1}\right)-s\left(x_{1}\right), \xi=(0,-1)^{\mathrm{T}}$. Now we can redefine

$$
\mathcal{U}=\left\{u \in\left(H^{1}(\Omega)\right)^{2} \mid u=0 \text { on } \Gamma_{u}, u_{2}\left(x_{1}, \alpha\left(x_{1}\right)\right) \geq-\alpha\left(x_{1}\right)+s\left(x_{1}\right), \quad \text { a.e. in }(a, b)\right\}
$$

and

$$
\begin{aligned}
\mathcal{U}_{m}=\left\{u_{m} \in(C(\bar{\Omega}))^{2}\left|u_{m}\right|_{\Omega^{e}} \in\left(P_{1}\right)^{2}, e=1, \ldots, n_{\mathrm{el}}, u_{m}=0 \text { on } \bar{\Gamma}_{u},\right. \\
\left.u_{2}\left(x_{1}^{j}, \alpha\left(x_{1}^{j}\right)\right) \geq-\alpha\left(x_{1}^{j}\right)+s\left(x_{1}^{j}\right), j=1, \ldots, n_{\mathrm{cn}}\right\} .
\end{aligned}
$$

Here $\left\{x_{1}^{j}\right\}_{j=1}^{n_{\mathrm{cn}}}$ denotes the finite sequence of $x_{1}$-coordinates of the contact nodes; cf. Fig. 2.

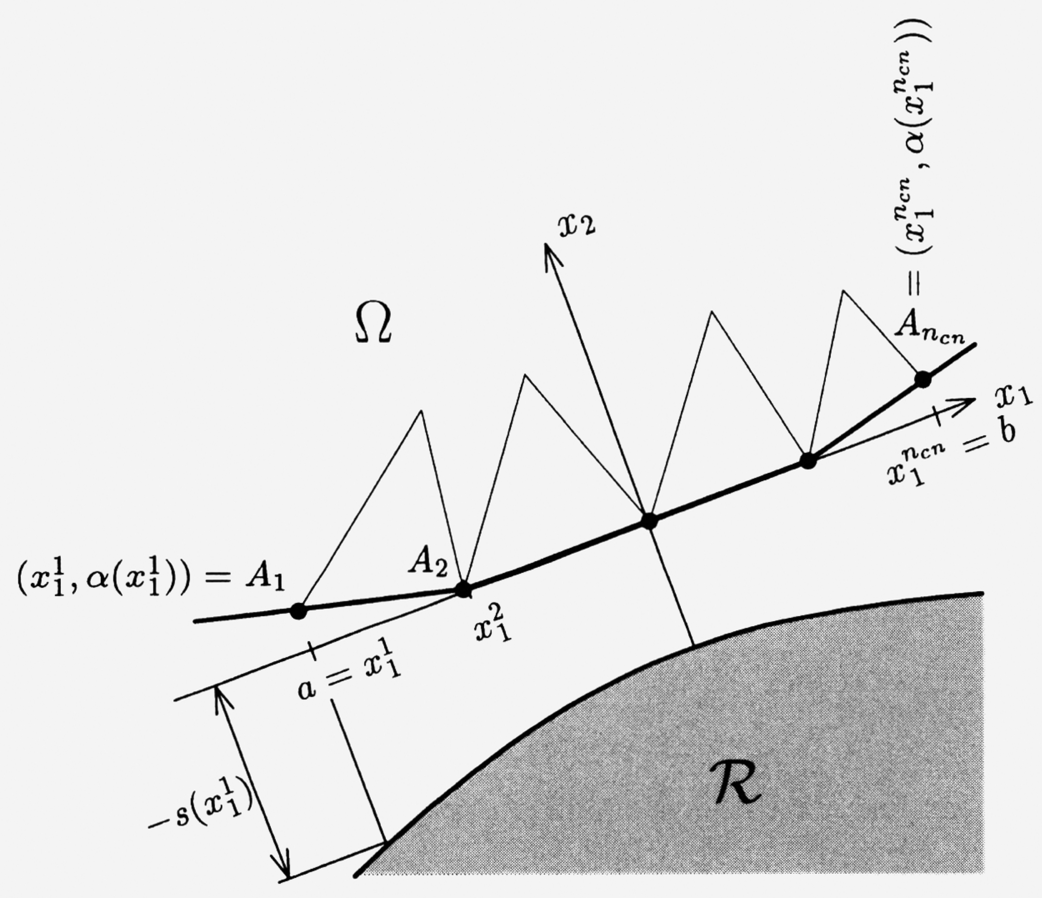

FIG. 2. Shapes of the potential contact boundaries

LEMMA 4.3. For any $u \in \mathcal{U}$ there exists a sequence $\left\{u^{\delta}\right\}, u^{\delta} \in \mathcal{U} \cap\left(C^{\infty}(\bar{\Omega})\right)^{2}$ such that

$$
u^{\delta} \stackrel{V}{\rightarrow} u \text { in } V, \quad \delta \rightarrow 0+,
$$

i.e., the set $\chi \equiv \mathcal{U} \cap\left(C^{\infty}(\bar{\Omega})\right)^{2}$ is dense in $\mathcal{U}$.

Proof. Let $u=\left(u_{1}, u_{2}\right)^{\mathrm{T}} \in \mathcal{U}$ be given. The approximation of the first component is standard: there is a sequence $\left\{u_{1}^{\delta}\right\}$ in $C^{\infty}(\bar{\Omega})$ such that

$$
u_{1}^{\delta}=0 \text { on } \Gamma_{u}, \quad u_{1}^{\delta} \rightarrow u_{1} \text { in } H^{1}(\Omega), \quad \delta \rightarrow 0+.
$$


Let us analyse the approximation of $u_{2}$. Define the function $\bar{u}_{2}$ as follows:

$$
\bar{u}_{2}\left(x_{1}, x_{2}\right)=\max \left\{u_{2}\left(x_{1}, x_{2}\right),-x_{2}+\tilde{s}\left(x_{1}\right)\right\}, \quad\left(x_{1}, x_{2}\right) \in \Omega
$$

Then $\bar{u}_{2} \in H^{1}(\Omega)$ and, since $\Gamma_{u} \subset \mathcal{Q}, \bar{u}_{2}=0$ on $\Gamma_{u}$. Moreover,

$$
\bar{u}_{2}\left(x_{1}, \alpha\left(x_{1}\right)\right)=u_{2}\left(x_{1}, \alpha\left(x_{1}\right)\right) \quad \text { a.e. in }(a, b) .
$$

We split $u_{2}$ as follows:

$$
u_{2}=\bar{u}_{2}+\Phi_{2}
$$

Then $\Phi_{2} \in H^{1}(\Omega), \Phi_{2}=0$ on $\Gamma_{c} \cup \Gamma_{u}$ as follows from (34). There exists a sequence $\left\{\Phi_{2}^{\delta}\right\}$, $\Phi_{2}^{\delta} \in C^{\infty}(\bar{\Omega})$ such that

$$
\begin{aligned}
& \operatorname{dist}\left(\operatorname{supp} \Phi_{2}^{\delta}, \bar{\Gamma}_{u} \cup \bar{\Gamma}_{c}\right)=\omega(\delta)>0, \\
& \Phi_{2}^{\delta} \rightarrow \Phi_{2} \quad \text { in } H^{1}(\Omega), \quad \delta \rightarrow 0+,
\end{aligned}
$$

where $\omega(\delta) \rightarrow 0+$ as $\delta \rightarrow 0+$. On the other hand, $\bar{u}_{2} \in H^{1}(\Omega), \bar{u}_{2}=0$ on $\Gamma_{u}$, and $\bar{u}_{2}\left(x_{1}, x_{2}\right) \geq-x_{2}+\tilde{s}\left(x_{1}\right),\left(x_{1}, x_{2}\right) \in \Omega$. From [17], page 38 (exercise 2.5) we know that there is another sequence $\left\{\bar{u}_{2}^{\delta}\right\}, \bar{u}_{2}^{\delta} \in C^{\infty}(\bar{\Omega}) \bar{u}_{2}^{\delta}=0$ on $\Gamma_{u}$ such that

$$
\begin{gathered}
\bar{u}_{2}^{\delta}\left(x_{1}, x_{2}\right) \geq-x_{2}+\tilde{s}\left(x_{1}\right) \quad \text { a.e. }\left(x_{1}, x_{2}\right) \in \Omega, \\
\bar{u}_{2}^{\delta} \rightarrow \bar{u}_{2} \quad \text { in } H^{1}(\Omega), \quad \delta \rightarrow 0+.
\end{gathered}
$$

Define

$$
u_{2}^{\delta} \equiv \bar{u}_{2}^{\delta}+\Phi_{2}^{\delta} \quad \forall \delta>0
$$

and

$$
u^{\delta} \equiv\left(u_{1}^{\delta}, u_{2}^{\delta}\right)^{\mathrm{T}} \quad \forall \delta>0
$$

Then $u^{\delta} \in \mathcal{U} \cap\left(C^{\infty}(\bar{\Omega})\right)^{2}$ and

$$
u^{\delta} \stackrel{V}{\rightarrow} u \quad \text { in } V, \quad \delta \rightarrow 0+
$$

due to (33), (36), and (38).

We choose $\pi_{m}$ to be the global (elementwise linear) Lagrange interpolation operator applied to each of the two components, and the rest of (jj) is therefore satisfied; cf. [15]. 


\section{Concluding remarks.}

5.1. The problem (Sח). It is rather straightforward to express the problem to solve, $(\mathrm{SJ})_{m}$, in matrix and vector terms. In fact, one arrives at

$$
\text { (Sח) }\left\{\begin{array}{l}
\text { Find }(\tilde{d}, \tilde{t}) \in \mathcal{K} \times \mathcal{W}: \\
\Pi(\tilde{d}, t) \leq \Pi(\tilde{d}, \tilde{t}) \leq \Pi(d, \tilde{t}), \quad \forall(d, t) \in \mathcal{K} \times \mathcal{W} .
\end{array}\right.
$$

A solution $\left(\tilde{u}_{m}, \tilde{h}_{m}\right) \in \mathcal{U}_{m} \times \mathcal{H}_{m}$ to $\left(\mathrm{SJ}_{m}\right)$ is obtained by setting $\tilde{u}_{m}=\mathcal{M}^{-1} \tilde{d}$ and $\tilde{h}_{m}=\mathcal{N}^{-1} \tilde{t}$. The function $\Pi$ is of the form

$$
\Pi(d, t)=\frac{1}{2} d^{\mathrm{T}} K(t) d-f^{\mathrm{T}} d
$$

where

$$
K(t)=\sum_{e=1}^{n_{\mathrm{el}}} t_{e} S_{e} K_{e}
$$

is the structural stiffness matrix and $K_{e}$ is the eth specific assembled element stiffness matrix. The vector $f$ is the structural load vector. The set of discretized thickness is of the form

$$
\mathcal{W}=\left\{t \in \mathbf{R}^{n_{\mathrm{el}}} \mid \underline{t} \leq t \leq \bar{t}, \sum_{e=1}^{n_{\mathrm{el}}} t_{e} S_{e}=V o l\right\} .
$$

We next treat the set $\mathcal{K}$, exclusively, in detail. We rewrite (30) and therefore study the term $u_{m}\left(A_{i}\right)^{\mathrm{T}} \xi$. Indeed, introducing (28) and (29),

$$
u_{m}\left(A_{i}\right)^{\mathrm{T}} \xi=\sum_{A=1}^{n_{\mathrm{np}}} N_{A}\left(A_{i}\right) d^{\mathrm{T}} C_{A} \xi .
$$

Denoting by $A(i)$ the node number in $\left\{1, \ldots, n_{\mathrm{np}}\right\}$ that has the coordinate $A_{i},(41)$ can be rewritten as

$$
u_{m}\left(A_{i}\right)^{\mathrm{T}} \xi=d^{\mathrm{T}} C_{A(i)} \xi
$$

using $N_{A}\left(A_{i}\right)=\delta_{A} A(i)$. Let us for $i=1, \ldots, n_{\mathrm{cn}}$ define vectors in $\mathbf{R}^{2 n_{\mathrm{np}}}$ :

$$
\nu_{i}=C_{A(i)} \xi
$$

so $(42)$ can be written as

$$
u_{m}\left(A_{i}\right)^{\mathrm{T}} \xi=\nu_{i}^{\mathrm{T}} d .
$$

Let $C$ be defined as the $\left(n_{\mathrm{cn}} \times 2 n_{\mathrm{np}}\right)$-matrix whose $i$ th row is $\nu_{i}^{\mathrm{T}}, i=1, \ldots, n_{\mathrm{cn}}$. Let also $q \in \mathbf{R}^{n_{\mathrm{cn}}}$ be defined through $q_{i}=g\left(A_{i}\right)$. Then (30) can be rewritten as

$$
\mathcal{U}_{m}=\left\{u_{m} \in V_{m} \mid \mathcal{M} u_{m} \in \mathcal{K}\right\}
$$

where now

$$
\mathcal{K}=\left\{d \in \mathbf{R}^{2 n_{\mathrm{np}}} \mid C d \leq q\right\} .
$$

Having (SI) to solve, one needs to know features of the matrices involved. The following hold:

- $K_{e}$ is symmetric for all $e=1, \ldots, n_{\mathrm{el}}$,

- $K_{e}$ is positive semidefinite for all $e=1, \ldots, n_{\mathrm{el}}$, 
- $K(t)$ is symmetric for any $t \in \mathbf{R}^{n_{\mathrm{el}}}$,

- $K(t)$ is positive semidefinite for all $t \in \mathbf{R}^{n_{\mathrm{el}}}, t \geq 0$,

- $K(t)$ is positive definite for any $t \in \mathbf{R}^{n_{\mathrm{el}}}, t>0$,

- $C$ is "quasi-orthogonal", i.e., the rows of $C$ are orthogonal.

The first five statements are standard, but we explicitly prove

THEOREM 5.1. $C C^{\mathrm{T}}=I$, where $I$ is the $n_{\mathrm{cn}} \times n_{\mathrm{cn}}$ unit matrix.

Proof. By (43) we have

$$
\left(C C^{\mathrm{T}}\right)_{i j}=\nu_{i}^{\mathrm{T}} \nu_{j}=\xi^{\mathrm{T}} C_{A(i)}^{\mathrm{T}} C_{A(j)} \xi .
$$

Whenever $i \neq j$ it can be seen that $C_{A(i)}^{\mathrm{T}} C_{A(j)}$ is the $2 \times 2$ zero matrix, while in the case $i=j$ it is the $2 \times 2$ unit matrix. If $i=j$,

$$
\left(C C^{\mathrm{T}}\right)_{i j}=\|\xi\|_{\mathbf{R}^{2}}^{2}=1
$$

and therefore $C C^{\mathrm{T}}=I$ follows.

The problem (Sח) is numerically solved in [18] by a subgradient optimization algorithm that relies on all the features listed above.

5.2. The abstract assumptions. Convergence analyses for displacement only equilibrium problems usually proceed in the following manner: The error (in norm) is estimated by using Céa's lemma (cf. [15]) and an interpolation error estimate for the conforming finite element at hand. Then convergence in norm is established as well as the rate of convergence, provided the solution is regular enough (as can sometimes be shown by using regularity theorems). In the case of contact, one cannot expect any additional regularity, and proceeds in a slightly different way, cf. [17]. In addition to the interpolation error estimate, one then needs to define a dense subset of regular functions $\chi$ included in the set of kinematically admissible displacements $\mathcal{U}, \mathrm{cf}$. (jj), and assume a weak closedness of the discretized version of $\mathcal{U}, \mathrm{cf}$. (j). These assumptions are used in this paper, as well as their counterparts for the "new" variable, i.e., (i) and (ii), and also the (non-standard) assumption (k). The assumption (i) is a simpler version of $(\mathbf{j})$, since it requires internal approximation, and in (ii) the requirement of a dense subset of regular functions has been dispensed with. The convergence indicated in (ii) is in the $L^{1}(\Omega)$ topology (since choosing the $L^{\infty}(\Omega)$-norm is quite obviously too strong), which is sufficient for our needs.

To convince the reader of the assumptions' simplicity, we indicate their verification in the non-contact case: The verification of (i), (ii), and (k) is not tedious as is evident from Sec. 4.1. For conforming finite elements, (j) follows immediately as well as (jj) by taking into account standard interpolation estimates and the fact that $C^{\infty}(\bar{\Omega})$ is dense in $H^{1}(\Omega)$. The length of Secs. 4.2 and 4.3 is due to the rather general contact situation.

As a consequence of the above description, any element that converges for the displacement analysis problem, in combination with elementwise constant thickness approximation, can be expected to satisfy the assumptions. If considering non-constant (continuous) design approximations however, it will be difficult to verify (ii) due to the constraints in the definition of $\mathcal{H}$.

5.3. Numerical instabilities and comparison with other saddle-point problems. In Theorem 3.1 we are only guaranteed an $L^{\infty}(\Omega)$ weak* convergence of the design variable, and 
hence checkerboard patterns or similar spurious modes in the design can appear. For criteria of (non-)presence of checkerboard formations, derived within discretized situations, we refer to [19] and [20].

In previous works on convergence associated with going from infinite dimension to discretized problems, it is usually one "crucial inequality" that implies the boundedness and uniqueness of a variable. The boundedness yields weak convergence and eventually, by using the crucial inequality, also strong convergence. In displacement analysis problems it is the bilinear form's ellipticity that is the crucial inequality, and in the Stokes' problem where the incompressibility condition is relaxed, the dual variable's crucial inequality is the LBB condition. The problem (SJ) seems to lack such a crucial inequality for the design variable, and instead the boundedness (and hence the $L^{\infty}(\Omega)$ weak* convergence) is given a priori. It is an open question if it is possible to prove strong convergence for the FE-discretized design in a suitable norm.

\section{REFERENCES}

[1] M. P. Rossow and J. E. Taylor, A finite element method for the optimal design of variable thickness sheets, AIAA J. 11, 1566-1568 (1973)

[2] J. Petersson, On stiffness maximization of variable thickness sheet with unilateral contact, Quart. Appl. Math. 54, 541-550 (1996)

[3] J. E. Taylor, Maximum strength elastic structural design, J. Engrg. Mech. Div., Proc. ASCE 95(EM3), 653-663 (1969)

[4] W. Prager and J. E. Taylor, Problems of optimal structural design, Trans. J. Appl. Mech. ASME 35 (1), 102-106 (1968)

[5] R. L. Benedict, Maximum stiffness design for elastic bodies in contact, J. Mech. Design 104, 825$830(1982)$

[6] J. Petersson, Stiffness optimization of general structure in Signorini-type contact, Contact Mechanics, edited by M. Raous, M. Jean and J. J. Moreau, Plenum Press, New York, 1995, pp. 41-48

[7] J. Céa and K. Malanowski, An example of a max-min problem in partial differential equations, SIAM J. Control 8, 305-316 (1970)

[8] M. P. Bendsøe and C. A. Mota Soares (editors), Topology Design of Structures, Kluwer Academic Publishers, Dordrecht, 1993

[9] M. P. Bendsøe, Optimization of Structural Topology, Shape, and Material, Springer-Verlag, Berlin, 1995

[10] F. Brezzi and M. Fortin, Mixed and Hybrid Finite Element Methods, Springer-Verlag, New York, 1991

[11] A. Klarbring, A. Mikelić, and M. Shillor, The rigid punch problem with friction, Internat. J. Engrg. Sci. 29, 751-768 (1991)

[12] N. Kikuchi and J. T. Oden, Contact Problems in Elasticity: A Study of Variational Inequalities and Finite Element Methods, SIAM, Philadelphia, 1988

[13] J. Haslinger and P. Neittaanmäki, Finite Element Approximation for Optimal Shape Design, John Wiley and Sons, London, 1988

[14] I. Hlaváček, J. Haslinger, J. Nečas, and J. Lovišek, Solution of Variational Inequalities in Mechanics, Springer-Verlag, New York, 1988

[15] P. G. Ciarlet, The Finite Element Method for Elliptic Problems, North-Holland Publishing Company, Amsterdam, 1978

[16] J. Haslinger, Finite element analysis for unilateral problems with obstacles on the boundary, Apl. Mat. 22, 180-187 (1977)

[17] R. Glowinski, Numerical Methods for Nonlinear Variational Problems, Springer Series in Computational Physics, Springer-Verlag, New York, 1984

[18] J. Petersson and M. Patriksson, Topology optimization of sheets in contact by a subgradient method, Internat. J. Numer. Methods Engrg. 40, 1295-1321 (1997) 
[19] C. S. Jog and R. B. Haber, Checkerboard and other spurious modes in solutions to distributedparameter and topology design problems, WCSMO-1: First World Congress of Structural and Multidisciplinary Optimization, edited by N. Olhoff and G. I. N. Rozvany, Elsevier Science Ltd., Oxford, 1995, pp. 237-242

[20] A. Diaz and O. Sigmund, Checkerboard patterns in layout optimization, Struct. Optim. 10, 40-45 (1995) 